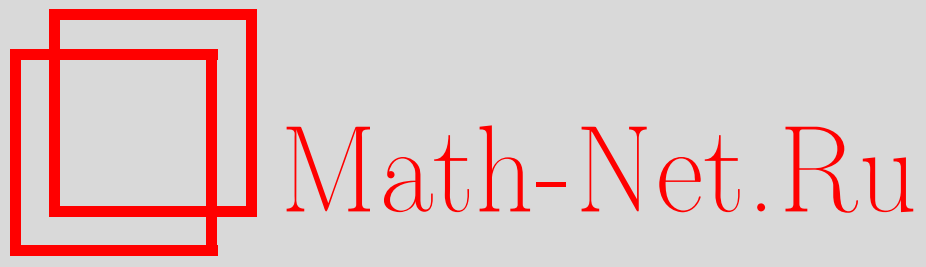

Х. Гаргуби, В. Ю. Овсиенко, Модули дифференциальных операторов на прямой, Функи. анализ и его прил., 2001, том 35, выпуск 1, 16-22

DOI: https://doi.org/10.4213/faa228

Использование Общероссийского математического портала MathNet.Ru подразумевает, что вы прочитали и согласны с пользовательским соглашением

http://www . mathnet.ru/rus/agreement

Параметры загрузки:

IP : 54.224 .135 .184

26 апреля 2023 г., 07:03:48

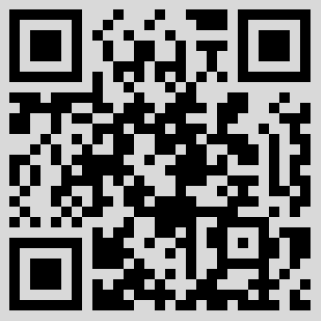


Функциональный анализ и его приложения

2001, т. 35, вып. 1, с. 16-22

УДК 517.9

\title{
Модули дифференциальных операторов на прямой
}

\author{
(c) 2001. Х. ГАРГУБИ, В. ОвСИЕНКо
}

1. Модули дифференциальных операторов. Обозначим через $\mathscr{D}^{k}$ пространство линейных дифференциальных операторов порядка $k$ на $\mathbb{R}$ (или на $S^{1}$ )

$$
A=A_{k}(x) \partial^{k}+A_{k-1}(x) \partial^{k-1}+\cdots+A_{0}(x), \quad \partial=\frac{d}{d x}, A_{i}(x) \in C^{\infty}(\mathbb{R}) .
$$

На пространстве $\mathscr{D}^{k}$ определено естественное 2 -параметрическое семейство модулей над группой диффеоморфизмов $\operatorname{Diff}(\mathbb{R})$ (и над алгеброй Ли векторных полей $\operatorname{Vect}(\mathbb{R}))$. Чтобы задать эту структуру, предположим, что дифференциальные операторы действуют на тензорных плотностях на $\mathbb{R}$, а именно, $A: \mathscr{F}_{\lambda} \rightarrow \mathscr{F}_{\mu}$, где $\mathscr{F}_{\lambda}$ обозначает $\operatorname{Diff}(\mathbb{R})$-модуль тензорных плотностей степени $-\lambda$, т. е. плотностей вида $\varphi(x)(d x)^{-\lambda}, \lambda \in \mathbb{R}\left(\right.$ см. [6]). Обозначим через $\mathscr{D}_{\lambda, \mu}^{k}$ модуль над $\operatorname{Diff}(\mathbb{R})$ $($ и над $\operatorname{Vect}(\mathbb{R}))$ дифференциальных операторов степени $k$ из $\mathscr{F}_{\lambda}$ в $\mathscr{F}_{\mu}$. Действие алгебры Ли $\operatorname{Vect}(\mathbb{R})$ на $\mathscr{D}_{\lambda, \mu}^{k}$ задано формулой

$$
L_{X}^{\lambda, \mu}(A)=L_{X}^{\mu} \circ A-A \circ L_{X}^{\lambda}, \quad X=X(x) \partial,
$$

где $L_{X}^{\lambda}=X \partial-\lambda X^{\prime}-$ оператор производной Ли на $\mathscr{F}_{\lambda}$ и где $X^{\prime}(x)=d X(x) / d x$.

На модуле $\mathscr{D}_{\lambda, \mu}$ всех дифференциальных операторов из $\mathscr{F}_{\lambda}$ в $\mathscr{F}_{\mu}$ определена естественная фильтрация $\mathscr{D}_{\lambda, \mu}^{0} \subset \mathscr{D}_{\lambda, \mu}^{1} \subset \cdots \subset \mathscr{D}_{\lambda, \mu}^{k} \subset \ldots$

Цель настоящей работы - классифицировать определенные выше модули: мы дадим полный список изоморфизмов между различными модулями $\mathscr{D}_{\lambda, \mu}^{k}$.

Проблема классификации модулей дифференциальных операторов на гладком многообразии была поставлена (в случае $\lambda=\mu$ ) и решена для модулей операторов второго порядка в [3]. Модули $\mathscr{D}_{\lambda, \lambda}^{k}$ на $\mathbb{R}$ (и на $S^{1}$ ) были классифицированы в [8]. В многомерном случае проблема классификации была недавно решена в $[12,16]$. Одномерный случай оказывается особенным и приводит к более интересным результатам.

2. Предварительные замечания. Заметим, прежде всего, что разность весов $\delta=\mu-\lambda$ является инвариантом, т. е. условие $\mathscr{D}_{\lambda, \mu}^{k} \cong \mathscr{D}_{\lambda^{\prime}, \mu^{\prime}}^{k}$ влечет за собой $\mu-\lambda=\mu^{\prime}-\lambda^{\prime}$. В самом деле, тот факт, что $\delta-$ инвариант, вытекает из условия эквивариантности по отношению к векторному полю $x \partial$.

Напомним также, что оператор сопряжения дифференциальных операторов определяет изоморфизм $\operatorname{Vect}(\mathbb{R})$-модулей $*: \mathscr{D}_{\lambda, \mu}^{k} \stackrel{\cong}{\longrightarrow} \mathscr{D}_{-1-\mu,-1-\lambda}^{k}$. Модуль из правой части этой формулы называется сопряженным модулем для $\mathscr{D}_{\lambda, \mu}^{k}$. Мы будем называть самосопряженными модули, для которых $\lambda+\mu=-1$ (т. е. стабильные по отношению к сопряжению). 
3. Классификационные результаты. Мы дадим здесь полную классификацию модулей $\mathscr{D}_{\lambda, \mu}^{k}$.

Tеорема 1. B случае $k \leqslant 3 \operatorname{Diff}(\mathbb{R})$-модули $\mathscr{D}_{\lambda, \mu}^{k}$ с фиксированными $k u \delta$ изоморфнь друг другу при всех значениях $\lambda(u \mu=\delta+\lambda)$, кроме модулей, заданных следующей таблицей:

\begin{tabular}{|c|c|c|c|}
\hline$k$ & 1 & 2 & 3 \\
\hline$(\lambda, \mu)$ & $(0,-1)$ & $(0, \mu)$ & $(0, \mu)$ \\
& & $(1 / 2,-3 / 2)$ & $(\lambda,-1-\lambda)$ \\
& & & $(\lambda,(4 \lambda-1) /(-3 \lambda+1))$ \\
& & & $(\lambda, \lambda-2)$ \\
\hline
\end{tabular}

а также сопряженных им модулей $\mathscr{D}_{-1-\mu,-1-\lambda}^{k}$.

Мы называем особыми модули $\mathscr{D}_{\lambda, \mu}^{k}$, которые не изоморфны никаким другим модулям, кроме сопряженных.

ТеОрема 2. Все модули, приведенные в таблицее (3), особые.

На следующем графике представлены все особые модули $\mathscr{D}_{\lambda, \mu}^{3}$.



Заметим, что прямая $\mu=-1$ соответствует модулям, сопряженным модулям с $\lambda=0$ из таблицы.

ЗАмЕЧАНИЕ. Наиболее известный классический пример модуля дифференциальных операторов - модуль операторов Штурма-Лиувилля $A=\partial^{2}+u(x)$ на $S^{1}$, действующих из $\mathscr{F}_{1 / 2}$ в $\mathscr{F}_{-3 / 2}$. Это - подмодуль самосопряженного модуля $\mathscr{D}_{1 / 2,-3 / 2}^{2}$. Заметим, что этот модуль связан с алгеброй Вирасоро (см. [10]). Мы надеемся, что другие особые модули, которые мы определили, тоже имеют интересную интерпретацию.

Сформулируем теперь наиболее общий результат.

ТЕОрема 3. При $k \geqslant 4$ не сущеетвует никаких изоморфизмов между различными $\operatorname{Diff}(\mathbb{R})$-модулями $\mathscr{D}_{\lambda, \mu}^{k}$, кроме сопряжения.

Заметим также, что в многомерном случае результат, аналогичный теореме 3 , верен при $k \geqslant 3$ (см. [12, 16]).

4. Модули символов. Пространство символов дифференциальных операторов (1) изоморфно пространству $\mathrm{Pol}^{k}\left(T^{*} \mathbb{R}\right)$ функций на $T^{*} \mathbb{R}$, полиномиальных 
$($ порядка $\leqslant k)$ на слоях. $\operatorname{Kak} \operatorname{Diff}(\mathbb{R})$ - (и $\operatorname{Vect}(\mathbb{R}))$-модуль пространство символов, соответствующее $\mathscr{D}_{\lambda, \mu}$, имеет вид

$$
\operatorname{Pol}\left(T^{*} \mathbb{R}\right) \otimes \mathscr{F}_{\delta} \cong \mathscr{F}_{\delta} \oplus \mathscr{F}_{\delta+1} \oplus \cdots \oplus \mathscr{F}_{\delta+k} \oplus \cdots, \quad \delta=\mu-\lambda .
$$

Для краткости мы будем использовать обозначение $\mathscr{S}_{\delta}^{k}$ вместо $\operatorname{Pol}^{k}\left(T^{*} \mathbb{R}\right) \otimes \mathscr{F} \delta$ и $\mathscr{S}_{\delta}$ для полного пространства символов.

Модуль символов изоморфен градуированному модулю, соответствующему фильтрованному модулю дифференциальных операторов, $\mathscr{S}_{\delta}=\operatorname{gr} \mathscr{D}_{\lambda, \mu}$.

5. Модули дифференциальных операторов над $\mathrm{sl}(2, \mathbb{R})$. Рассмотрим подалгебру Ли $\operatorname{sl}(2, \mathbb{R}) \subset \operatorname{Vect}(\mathbb{R})$ с базисом $\left\{\partial, x \partial, x^{2} \partial\right\}$ и ограничим на нее действие (2). Эта алгебра Ли играет специальную роль, она позволяет отождествить пространства $\mathscr{D}_{\lambda, \mu}^{k}$ и $\mathscr{S}_{\delta}^{k}$, где $\delta=\mu-\lambda$, каноническим образом.

Следующий результат показывает, что для неособых значений $\delta$ пространство $\mathscr{D}_{\lambda, \mu}^{k}$ изоморфно $\mathscr{S}_{\delta}^{k}$ как $\mathrm{sl}(2, \mathbb{R})$-модуль.

Tеорема 4. (i) Если $\delta \neq-1,-3 / 2,-2,-5 / 2, \ldots,-k$, то $\mathscr{D}_{\lambda, \mu}^{k}$ изоморфно $\mathscr{S}_{\delta}^{k}$ $\kappa a \kappa \operatorname{sl}(2, \mathbb{R})-$ модуль.

(ii) Существует единственньй изоморфизм sl $(2, \mathbb{R})$-модулей $\sigma: \mathscr{D}_{\lambda, \mu}^{k} \rightarrow \mathscr{S}_{\delta}^{k}$, сохраняющий главный символ дифференциального оператора.

Изоморфизм $\sigma$ называется проективно эквивариантным символом. Нам понадобится явная формула, заимствованная из [2] (см. также [11, 13] в многомерном случае). Отображение $\sigma$ переводит однородный дифференциальный оператор $A=A_{k}(x) \partial^{k}$ порядка $k$ в полиномиальную функцию $\sigma(A)=\sum_{i=0}^{k} \alpha_{i}^{k} A_{k}^{(i)} \xi^{i}$, где $A_{k}^{(i)}=\partial^{i} A_{k}$ и константы $\alpha_{i}^{k}$ заданы формулой

$$
\alpha_{i}^{k}=\frac{\left(\begin{array}{c}
k \\
i
\end{array}\right)\left(\begin{array}{c}
2 \lambda-k+i \\
2 \lambda-k
\end{array}\right)}{\left(\begin{array}{c}
2 \delta+2 k-i+1 \\
2 \delta+2 k-2 i+1
\end{array}\right)}
$$

Особые значения $\delta$, для которых знаменатель в (5) обращается в нуль, называются резонансами. В этом случае оператор Казимира алгебры $\operatorname{sl}(2, \mathbb{R})$ на модуле $\mathscr{D}_{\lambda, \mu}$ имеет кратные собственные значения (см. [4]).

Сформулируем результат в резонансном случае.

ПрЕДЛОЖЕНИЕ 1. При $\delta=-1,-3 / 2,-2,-5 / 2, \ldots,-k$ изоморфизм $\operatorname{sl}(2, \mathbb{R})$-модулей б существует, если и только если

$$
(\lambda, \mu)= \begin{cases}(\ell / 2,-(\ell+2) / 2), & 0 \leqslant \ell \leqslant k-1, \\ (\ell / 2,-(\ell+1) / 2) \text { или }((\ell-1) / 2,-(\ell+1) / 2), & 1 \leqslant \ell \leqslant k-1 .\end{cases}
$$

В этом случае, однако, изоморфизм $\mathrm{sl}(2, \mathbb{R})$-модулей не является единственным.

Основная идея доказательства теорем 1 и 3 - использовать $\operatorname{sl}(2, \mathbb{R})$-эквивариантный символ, чтобы привести действие алгебры Ли $\operatorname{Vect}(\mathbb{R})$ на $\mathscr{D}_{\lambda, \mu}$ к каноническому виду. Другими словами, мы будем использовать следующую диаграмму:

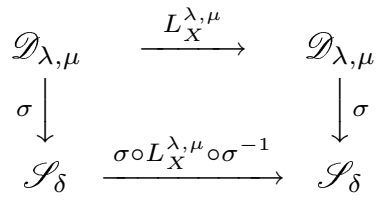


и сравнивать действие $\sigma \circ L_{X}^{\lambda, \mu} \circ \sigma^{-1}$ со стандартным действием алгебры Ли $\operatorname{Vect}(\mathbb{R})$ на $\mathscr{S}_{\delta}$.

6. Действие алгебры Ли $\operatorname{Vect}(\mathbb{R})$ в $\operatorname{sl}(2, \mathbb{R})$-инвариантной форме. Мы вычислим действие алгебры Ли $\operatorname{Vect}(\mathbb{R})$ на $\mathscr{D}_{\lambda, \mu}^{k}$ при $k \leqslant 4$ в терминах $\operatorname{sl}(2, \mathbb{R})$ эквивариантного символа.

Простое вычисление приводит к следующей формуле: действие алгебры Ли $\operatorname{Vect}(\mathbb{R})$ на пространстве полиномиальных функций $P=P_{4} \xi^{4}+\cdots+P_{0}$ степени 4 , заданное выражением $\sigma \circ L_{X}^{\lambda, \mu} \circ \sigma^{-1}$, имеет вид $\sigma \circ L_{X}^{\lambda, \mu} \circ \sigma^{-1}(P)=P_{4}^{X} \xi^{4}+\cdots+$ $P_{0}^{X}$, где

$$
\begin{aligned}
& P_{4}^{X}=L_{X}^{\delta+4}\left(P_{4}\right), \\
& P_{3}^{X}=L_{X}^{\delta+3}\left(P_{3}\right), \\
& P_{2}^{X}=L_{X}^{\delta+2}\left(P_{2}\right)+\beta_{2}^{4} J_{3}\left(X, P_{4}\right), \\
& P_{1}^{X}=L_{X}^{\delta+1}\left(P_{1}\right)+\beta_{1}^{3} J_{3}\left(X, P_{3}\right)+\beta_{1}^{4} J_{4}\left(X, P_{4}\right), \\
& P_{0}^{X}=L_{X}^{\delta}\left(P_{0}\right)+\beta_{0}^{2} J_{3}\left(X, P_{2}\right)+\beta_{0}^{3} J_{4}\left(X, P_{3}\right)+\beta_{0}^{4} J_{5}\left(X, P_{4}\right) ;
\end{aligned}
$$

здесь $\beta_{i}^{j}$ - числа, заданные формулой (9) ниже, а билинейные операторы $J_{\ell}\left(X, P_{k}\right)$ - так называемые трансвектанты, т. е. единственные (с точностью до константы $\operatorname{sl}(2, \mathbb{R})$-эквивариантные билинейные операторы на тензорных плотностях (см. [9] и цитируемую там литературу).

В нашем случае

$$
\begin{aligned}
& J_{3}\left(X, P_{s}\right)=X^{\prime \prime \prime} P_{s}, \\
& J_{4}\left(X, P_{s}\right)=s X^{(I V)} P_{s}+2 X^{\prime \prime \prime} P_{s}^{\prime}, \\
& J_{5}\left(X, P_{s}\right)=s(2 s-1) X^{(V)} P_{s}+5(2 s-1) X^{(I V)} P_{s}^{\prime}+10 X^{\prime \prime \prime} P_{s}^{\prime \prime}
\end{aligned}
$$

для $s=2,3,4$.

Заметим, что коэффициенты при старших степенях, $P_{4}$ и $P_{3}$, преобразуются как тензорные плотности; при преобразовании коэффициентов при более низких степенях возникают дополнительные члены, что различает действие $\sigma \circ L_{X}^{\lambda, \mu} \circ \sigma^{-1}$ и стандартное действие алгебры Ли $\operatorname{Vect}(\mathbb{R})$ на $\mathscr{S}_{\delta}$.

Числовые коэффициенты $\beta_{i}^{j}$ в $(7)$ заданы формулой

$$
\begin{aligned}
& \beta_{2}^{4}(\lambda, \mu)=\frac{(6 \lambda-4) \delta+6 \lambda^{2}+6 \lambda-5}{7+2 \delta} \\
& \beta_{1}^{3}(\lambda, \mu)=\frac{(3 \lambda-1) \delta+3 \lambda^{2}+3 \lambda-1}{5+2 \delta} \\
& \beta_{1}^{4}(\lambda, \mu)=\frac{(\delta+2 \lambda+1)[(4 \lambda-1) \delta+4 \lambda(\lambda+1)]}{(2+\delta)(3+\delta)(4+\delta)} \\
& \beta_{0}^{2}(\lambda, \mu)=\frac{\lambda(\delta+\lambda+1)}{3+2 \delta}, \quad \beta_{0}^{3}(\lambda, \mu)=\frac{\lambda(\delta+\lambda+1)(\delta+2 \lambda+1)}{(1+\delta)(2+\delta)(3+\delta)} \\
& \beta_{0}^{4}(\lambda, \mu)=\frac{\lambda(\delta+\lambda+1)\left(4 \delta^{2}+12 \lambda \delta+12 \delta+12 \lambda^{2}+12 \lambda+11\right)}{(1+\delta)(3+2 \delta)(5+2 \delta)(7+2 \delta)(4+\delta)}
\end{aligned}
$$


Заметим, что резонансные значения $\delta$ из теоремы 4 и предложения 1 - это в точности те значения, для которых коэффициенты (9) не определены.

7. Конструкция изоморфизма. Мы дадим здесь схему доказательства теорем 1 и 3. Подробности и вычисления будут опубликованы отдельно.

Для доказательства теоремы 1 мы построим явно искомый изоморфизм в терминах проективно эквивариантного символа. Положим $P_{4} \equiv 0$ и рассмотрим линейное отображение $T: \mathscr{D}_{\lambda, \mu}^{3} \rightarrow \mathscr{D}_{\lambda^{\prime}, \mu^{\prime}}^{3}$, заданное формулой

$$
T\left(P_{3} \xi^{3}+\cdots+P_{0}\right)=P_{3} \xi^{3}+\frac{{\beta_{0}^{\prime}}_{0}^{3} \beta_{0}^{2}}{\beta_{0}^{3} \beta_{0}^{\prime 2}} P_{2} \xi^{2}+\frac{{\beta_{1}^{\prime}}_{1}^{3}}{\beta_{1}^{3}} P_{1} \xi^{1}+\frac{{\beta_{0}^{\prime}}_{0}^{3}}{\beta_{0}^{3}} P_{0},
$$

где ${\beta^{\prime}}_{i}^{j}=\beta_{i}^{j}\left(\lambda^{\prime}, \mu^{\prime}\right)-$ коэффициенты действия (7). Это отображение оказывается изоморфизмом $\operatorname{Vect}(\mathbb{R})$-модулей. В самом деле, легко проверить, что это отображение перестановочно с действием алгебры Ли $\operatorname{Vect}(\mathbb{R})$ на $\mathscr{D}_{\lambda, \mu}^{3}$ и $\mathscr{D}_{\lambda^{\prime}, \mu^{\prime}}^{3}$. Это доказывает теорему 1 для нерезонансных значений $\delta$ (т. е. для $\delta \neq-1,-3 / 2,-2$, $-5 / 2,-3)$, поскольку мы использовали проективно эквивариантный символ при построении изоморфизма.

Изоморфизм (10), тем не менее, имеет смысл для резонансных $\delta$, что завершает доказательство теоремы 1. Чтобы проверить это, достаточно переписать формулу (10) в терминах коэффициентов дифференциальных операторов (1). Мы не приводим здесь это прямое вычисление.

Рассмотрим теперь изоморфизм $T: \mathscr{D}_{\lambda, \mu}^{k} \rightarrow \mathscr{D}_{\lambda^{\prime}, \mu^{\prime}}^{k}$, где $k \geqslant 4$. Поскольку $T$ - изоморфизм $\operatorname{Vect}(\mathbb{R})$-модулей, он также является изоморфизмом $\operatorname{sl}(2, \mathbb{R})$-модулей. Единственность $\mathrm{sl}(2, \mathbb{R})$-эквивариантного символа показывает, что линейное отображение $\sigma \circ T \circ \sigma^{-1}$ на $\mathscr{S}_{\delta}^{k}$ диагонально и состоит в умножении на константу каждой однородной компоненты (так же, как изоморфизм (10) выше).

Легко показать (см. [12]), что ограничение $T$ на $\mathscr{D}_{\lambda, \mu}^{4} \subset \mathscr{D}_{\lambda, \mu}^{k}$ должно быть изоморфизмом на подмодуль $\mathscr{D}_{\lambda^{\prime}, \mu^{\prime}}^{4}$. Предполагая опять, что $\delta$ нерезонансно, так что существует проективно эквивариантный символ, можно проверить, что линейное отображение $T\left(P_{4} \xi^{4}+\cdots+P_{0}\right)=P_{4} \xi^{4}+\tau_{3} P_{3} \xi^{3}+\cdots+\tau_{0} P_{0}$ с неопределенными $\tau_{i}$, зависящее от $\lambda, \mu, \lambda^{\prime}, \mu^{\prime}$, перестановочно с действием алгебры Ли $\operatorname{Vect}(\mathbb{R})$, заданным формулами $(7)$ на $\mathscr{D}_{\lambda, \mu}^{4}$ и $\mathscr{D}_{\lambda^{\prime}, \mu^{\prime}}^{4}$, если и только если

$$
\begin{aligned}
& \tau_{4}{\beta^{\prime}}_{2}^{4}=\tau_{2} \beta_{2}^{4}, \quad \tau_{4}{\beta^{\prime}}_{1}^{4}=\tau_{1} \beta_{1}^{4}, \quad \tau_{4}{\beta^{\prime}}_{0}^{4}=\tau_{0} \beta_{0}^{4}, \\
& \tau_{3}{\beta^{\prime}}^{3}=\tau_{1} \beta_{1}^{3}, \quad \tau_{3}{\beta^{\prime}}_{0}^{3}=\tau_{0} \beta_{0}^{3}, \quad \tau_{2}{\beta^{\prime 2}}_{0}^{2}=\tau_{0} \beta_{0}^{2} .
\end{aligned}
$$

Эта система имеет решение только при $\lambda=\lambda^{\prime}$ или $\lambda+\mu^{\prime}=-1$. Первый изоморфизм тривиален, а второй в точности совпадает с сопряжением. Это доказывает теорему 3 для нерезонансных $\delta$.

Мы не приводим вычисления для резонансного случая.

8. Когомологии алгебры Ли $\operatorname{Vect}(\mathbb{R})$, связанные с модулями $\mathscr{D}_{\boldsymbol{\lambda}, \boldsymbol{\mu}}^{\boldsymbol{k}}$. Тот факт, что фильтрованный модуль $V$ над алгеброй Ли g можно рассматривать как деформацию соответствующего градуированного модуля $\operatorname{gr} V$, является общим. Эта деформация связана с первой группой когомологий с коэффициентами в End $(\operatorname{gr} V)$. Мы ссылаемся здесь на классическую теорию Ричардсона-Ниенхейса $[17,18]$. 
Модуль $\mathscr{D}_{\lambda, \mu}$ является, стало быть, нетривиальной деформацией модуля символов $\mathscr{S}_{\delta}$ с $\mu-\lambda=\delta$ (см. $\left.[3,8,14]\right)$. Этот модуль связан с пространством когомологий $H^{1}\left(\operatorname{Vect}(\mathbb{R}) ; \operatorname{End}\left(\mathscr{S}_{\delta}\right)\right)=\bigoplus_{k>\ell} H^{1}\left(\operatorname{Vect}(\mathbb{R}) ; \operatorname{Hom}\left(\mathscr{F}_{\delta+k}, \mathscr{F}_{\delta+\ell}\right)\right)$. Точнее, трансвектанты $J_{\ell}\left(X, P_{s}\right)$ с $\ell=3,4,5$ определяют нетривиальнье 1-коцикльь на $\operatorname{Vect}(\mathbb{R})$ со значениями в $\operatorname{Hom}\left(\mathscr{F}_{\delta+s}, \mathscr{F}_{\delta+s-\ell+1}\right)$ :

$$
C_{\ell}(X)=J_{\ell}(X, \cdot)
$$

$($ cм. [8]). Действие алгебры Ли $\operatorname{Vect}(\mathbb{R})$, заданное формулой $(7)$, - нетривиальная деформация действия на пространстве символов.

ЗАмЕЧАНИЕ. Пространство когомологий $H^{1}\left(\operatorname{Vect}(\mathbb{R}) ; \operatorname{Hom}\left(\mathscr{F}_{\nu}, \mathscr{F}_{\nu^{\prime}}\right)\right)$ было определено в [5] для алгебры Ли полиномиальных векторных полей на $\mathbb{R}$; см. также [15] для случая полиномиальных векторных полей на $S^{1}$. Для алгебры Ли гладких векторных полей соответствующее пространство было описано в [14]; см. также [1].

Мы будем использовать нетривиальные коциклы (11) при доказательстве теоремы 2. Тот факт, что эти коциклы нетривиальны, влечет за собой существование особых модулей, если хоть один из коэффициентов $\beta_{i}^{j}$ в (7) обращается в нуль. Теорема 2 следует теперь из явных формул (9).

ЗАмечАниЕ. Ограничения коциклов $C_{\ell}(X)$ при $\ell=3,4,5$ на $\operatorname{sl}(2, \mathbb{R})$ обращаются в нуль и задают нетривиальные классы так называемых относительных когомологий $H^{1}\left(\operatorname{Vect}\left(S^{1}\right), \operatorname{sl}(2, \mathbb{R}) ; \operatorname{Hom}\left(\mathscr{F}_{\lambda}, \mathscr{F}_{\mu}\right)\right)$.

9. Препятствия к существованию $\operatorname{sl}(2, \mathbb{R})$-эквивариантного символа. Для резонансных значений $\delta=-1,-3 / 2,-2,-5 / 2, \ldots,-k$ существует серия классов когомологий $\mathrm{sl}(2, \mathbb{R})$, являющихся препятствиями к существованию изоморфизма из теоремы 4. Более точно, рассмотрим линейное отображение $C_{2}^{n}: \operatorname{sl}(2, \mathbb{R}) \rightarrow$ $\operatorname{Hom}\left(\mathscr{F}_{n / 2}, \mathscr{F}_{-1-n / 2}\right)$, заданное формулой

$$
C_{2}^{n}(X)\left(a(d x)^{-n / 2}\right):=X^{\prime \prime} a^{(n)}(d x)^{1+n / 2} .
$$

Легко проверить (см. [7]), что это отображение - нетривиальный 1-коцикл на $\operatorname{sl}(2, \mathbb{R})$ для каждого $n \in \mathbb{N}$. Эти коциклы возникают при действии алгебры Ли $\operatorname{sl}(2, \mathbb{R})$ на $\mathscr{D}_{\lambda, \mu}$, заданном формулой $(2)$.

Можно, тем не менее, определить канонический символ в резонансном случае так, чтобы мерой его $\mathrm{sl}(2, \mathbb{R})$-неэквивариантности служил коцикл (12). После этого доказательство теорем 1 и 3 для резонансного случая повторяет доказательство для общего случая.

ЗАмЕЧАНИЕ. (а) Пространство когомологий $\operatorname{sl}(n+1, \mathbb{R})$ с коэффициентами в $\operatorname{Hom}\left(\mathscr{S}_{\delta}^{k}, \mathscr{S}_{\delta}^{\ell}\right)$ было полностью вычислено в [11]. В этой работе были также получены многомерные аналоги теоремы 4 и предложения 1.

(б) Фактормодули $\mathscr{D}_{\lambda, \lambda}^{k} / \mathscr{D}_{\lambda, \lambda}^{\ell}$ при $k>\ell$ были классифицированы в [13].

(в) Пространство $H^{1}\left(\operatorname{Vect}\left(S^{1}\right), \operatorname{sl}(2, \mathbb{R}) ; \mathscr{D}_{\lambda, \mu}\right)$ относительных когомологий $\operatorname{Vect}\left(S^{1}\right)$ (т. е. когомологий комплекса коцепей на $\operatorname{Vect}(\mathbb{R})$, обращающихся в нуль на $\operatorname{sl}(2, \mathbb{R}))$ было впервые рассмотрено в [8] и полностью описано в $[1,14]$.

Мы благодарны К. Дювалю, Б. Л. Фейгину и П. Леконту за полезные обсуждения. 


\section{ЛИТЕРАТУРА}

1. Bouarroudj S., Ovsienko $V$. Three cocycles on $\operatorname{Diff}\left(S^{1}\right)$ generalizing the Schwarzian derivative. Internat. Math. Res. Notices, No. 1, 25-39 (1998).

2. Cohen P., Manin Yu., Zagier D. Automorphic pseudodifferential operators. In: Progr. Nonlinear Diff. Eq. Appl., Vol. 26, Birkhäuser, Boston, 1997, pp. 17-47.

3. Duval C., Ovsienko V. Space of second order linear differential operators as a module over the Lie algebra of vector fields. Adv. in Math., 132, No. 2, 316-333 (1997).

4. Duval C., Lecomte P., Ovsienko $V$. Conformally equivariant quantization: existence and uniqueness. Ann. Inst. Fourier, 49, No. 6, 1999-2029 (1999).

5. Фейгин Б. Л., Фукс Д. Б. Гомологии алгебры Ли векторных полей на прямой. Функц. анализ и его прил., 14, No. 3, 45-60 (1980).

6. Фукс Д. Б. Когомологии бесконечномерных алгебр Ли. Наука, М., 1984.

7. Gargoubi H. Modules des opérateurs différentiels sur la droite: géométrie projective et cohomologie de Gelfand-Fuchs. Thesis, Université de Provence, 1997.

8. Gargoubi H., Ovsienko $V$. Space of linear differential operators on the real line as a module over the Lie algebra of vector fields. Internat. Math. Res. Notices, No. 5, 235251 (1996).

9. Janson S., Peetre J. A new generalization of Hankel operators (the case of higher weights). Math. Nachr., 132, 313-328 (1987).

10. Kirillov A. A. Infinite dimensional Lie groups: their orbits, invariants and representations. The geometry of moments. In: Twistor geometry and nonlinear systems, Lect. Notes in Math., Vol. 970, 1982, pp. 101-123.

11. Lecomte P. B. A. On the cohomology of $\operatorname{sl}(m+1, \mathbb{R})$ acting on differential operators and $\operatorname{opsl}(m+1, \mathbb{R})$-equivariant symbol. Preprint Université de Liège, 1998.

12. Lecomte P. B. A., Mathonet P., Tousset E. Comparison of some modules of the Lie algebra of vector fields. Indag. Math., N.S., 7, No. 4, 461-471 (1996).

13. Lecomte P. B. A., Ovsienko V. Projectively invariant symbol calculus. Lett. Math. Phys., 49, No. 3, 173-196 (1999).

14. Lecomte P. B. A., Ovsienko V. Cohomology of the Vector fields Lie algebra and modules of differential operators on a smooth manifold. Comp. Math., 124, No. 1, 95-110 (2000).

15. Martin C., Piard A. Classification of the indecomposable bounded admissible modules over the Virasoro Lie algebra with weightspaces of dimension not exceeding two. Comm. Math. Phys., 150, No. 3, 465-493 (1992).

16. Mathonet $P$. Intertwining operators between some spaces of differential operators on a manifold. Comm. Algebra, 27, No. 2, 755-776 (1999).

17. Nijenhuis A., Richardson R. W. Deformations of homomorphisms of Lie algebras. Bull. Amer. Math. Soc., 73, 175-179 (1967).

18. Richardson $R$. W. Deformations of subalgebras of Lie algebras. J. Diff. Geom., 3, 289-308 (1969).

Département de Mathématiques, IPEIM, Monastir, Tunisie e-mail: Hichem.Gargoubi@ipeim.rnu.tn

CNRS, Centre de Physique Théorique,

Luminy Case 907, Marseille, France

Поступило в редакцию 4 октября 1999 г.

e-mail: Valentin.Ovsienko@cpt.univ-mrs.fr

В переработанном виде 11 мая 2000 г. 